\title{
A Simple Bed-side Method of Enteral Feeding through Distal Mucous Fistula in Patients with Short Bowel Syndrome to overcome Parenteral Dependency: The Jategaonkar Technique
}

\author{
Priyadarshan Anand Jategaonkar1 and Sudeep Pradeep Yadav² \\ IDepartment of Surgery, Division of GI and Minimal Access Surgery, Mahatma Gandhi Institute of Medical Sciences, Sevagram, \\ Wardha, Maharashtra, India \\ 2Department of Plastic and Reconstructive Surgery, Grant's Government Medical College and Sir J.J. Group of Hospitals, J.J. Marg, \\ Nagpada, Mumbai Central, Mumbai, Maharashtra, India
}

\begin{abstract}
Today, massive proximal small bowel resection with diverting stoma formation is a relatively common occurrence, especially in an emergency setting. However, the resultant short bowel syndrome remains difficult to manage on various nutritional fronts and commencing total parenteral nutrition, along with plethora of its associated complications, becomes almost obligatory for these unfortunate patients. In this context, the authors describe an innovative, yet, handy method of enteral feeding through distal mucous fistula using two commonly available tubes in the ward, with the aim to maximise the usage of the available gut and to outwit the ensuing need of parenteral supplementation, and name it the Jategaonkar technique. Backed with successful experience of 18 cases, it is especially useful in low-income countries and can be mastered readily even by junior doctors, paramedic staff, and stoma therapists or by patient's kin, alike. Such a procedure is yet to be reported in the available literature.
\end{abstract}

Key Words: Short bowel syndrome, Enteral feeding, Massive bowel resections, Distal mucous fistula, Jejunostomy re-feeding, Parenteral nutrition.

How to cite this article: Jategaonkar PA, Yadav SP. A simple bed-side method of enteral feeding through distal mucous fistula in patients with short bowel syndrome to overcome parenteral dependency: The Jategaonkar technique. J Coll Physicians Surg Pak 2019; 29(12):1212-1214.

\section{INTRODUCTION}

Owing to a substantial trophic effects, enteral route has been adjudged better than the parenteral route in critically ill patients. ${ }^{1,2}$ However, in a unique surgical scenario, wherein a patient undergoes major emergent small bowel resection with proximal jejunostomy (PJ) and distal ileal mucous fistula (MF), it becomes increasingly difficult to optimise nutrition without having to resort to commercial parenteral preparations. Herein, high output PJ invariably leads to unstable homeostasis which could eventually lead to loss of life. Yet, for these seriously ill individuals, the ideal and most sought-after route for continued nutritional support remains enteral. ${ }^{3}$ In this perspective, we present an easy-to-practice, safe and inexpensive system of tube-mucofistuloclysis using a unique symbiotic assembly of Ryle's naso-gastric tube (RT) and Foley's urinary catheter (FC) and name it the Jategaonkar technique.

Correspondence to: Prof. Priyadarshan Anand Jategaonkar, Department of Surgery, Division of GI and Minimal Access Surgery, Mahatma Gandhi Institute of Medical Sciences,

Sevagram, Wardha-442102, Maharashtra, India

E-mail: jategaonkarpa@gmail.com

Received: February 28, 2019; Revised: April 22, 2019;

Accepted: May 20, 2019

\section{TECHNIQUE}

A combination is standard RT (16 Fr for adults and $12 \mathrm{Fr}$ for pediatric patients) and FC (14 Fr) is used. Once MF is matured, a gloved index finger (for adults and little finger for pediatric patients) smeared with lignocain jelly or paraffin is gently inserted into it for noting the level of rent in the abdominal musculature which is often felt convincingly as a firm circumferential ridge. Now, the RT is inserted gradually up to the second mark and secured to the abdominal skin with $2 / 0$ silk with its infusion knob closed. A vaseline-gauze dressing is then applied to the MF before shifting the patient out. At the appropriate clinical time of commencing the enteral feeds in the postoperative period, a FC is introduced into the MF and held (and not sutured to the skin) steady in such a way that its balloon segment rests at the pre-judged level of anterior abdominal wall and is readily visible from externally. At this time, to make it self-retaining, the balloon is gradually inflated with $5-10 \mathrm{ml}$ water and is then clamped to prevent the backflow of enteric contents (Figure 1). Also, its strategic position blocks the enteral lumen completely. Now, patency of the RT is confirmed by flushing it with normal saline and then the contemplated feed is infused through it at a predetermined dose and rate. Post feed, the FC balloon is continued to be kept inflated for 30 more minutes, then 


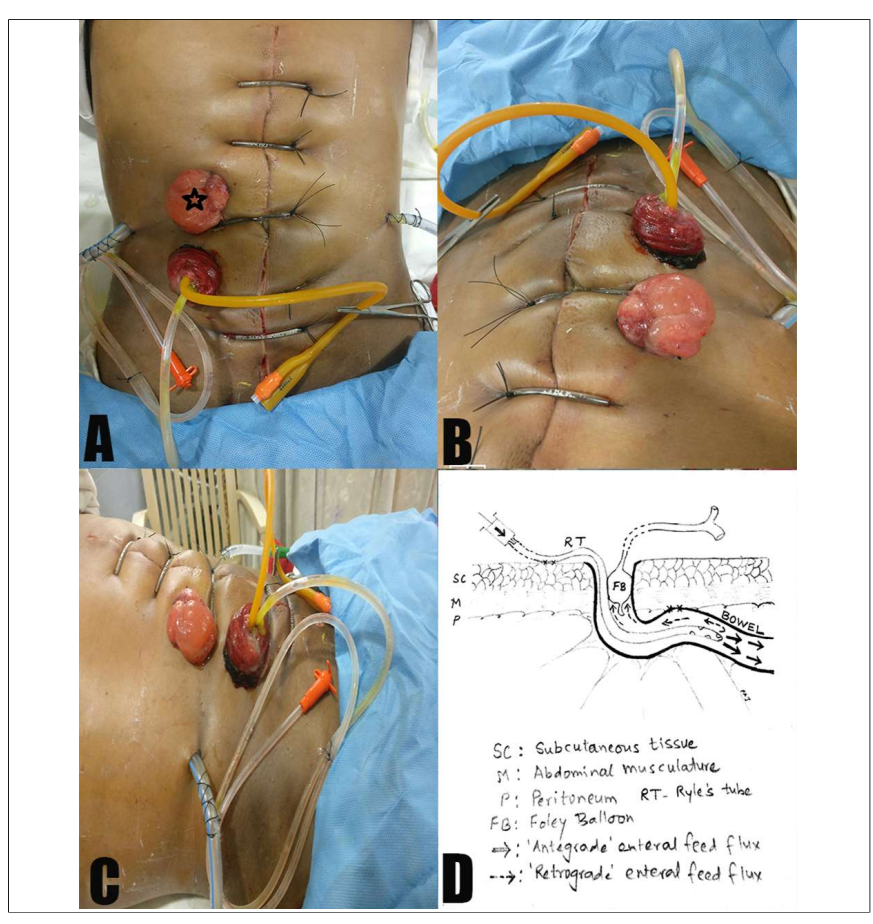

Figure 1: The system of double-tube mucofistuloclysis. Snaps A-C illustrates the typical positions of the Ryle's tube and the Foley catheter inserted into the mucous fistula before commencing the enteral feed in the ward. The proximal stoma $\left({ }^{*}\right)$ looks edematous but healthy. Schema D depicts the luminal play of enteric currents during the feed-infusion cycles. Note that the strategically placed Foley balloon acts as a "gatekeeper" to maximise function of the redundant bowel.

deflated and removed to conclude the procedure. These steps are repeated sequentially for all the subsequent feeding cycles.

\section{DISCUSSION}

Though enteral re-feeding has come a long way since 1970s, modern literature enigmatically continues to lack conclusive ways to accomplish it satisfactorily on a larger scale. Over these four-and-half decades, for a small but significant subset of patients suffering critical loss of intestinal mass due to diverse reasons, various investigators have discovered that timely reinfusion of PJ effluent into the MF maintains the requisite nutritional level to the extent of abolishing any further need for parental supplementation.2-4 However, the hitherto available options for such mucofistuloclysis seem not only clumsy and expensive but potentially lifethreatening as well.3-6 This could be of great concern, especially for resource-limited countries.

In this perspective, the presently described technique has following advantages. First, the assembly is easy to reproduce and quick to employ (our average time for setting it up remains 3 minutes). Also, it can commence functioning in the immediate postoperative period. It uses readily available resources that tend to make it a low-cost option. The inflated balloon recycles the administered feeds repeatedly through the available distal small intestinal segment, thus, maximising its use for digestion and absorption of nutrients, electrolytes and minerals. FC balloon also prevents retrograde spillage of the feeds and the resultant peristomal skin excoriation. In an effort to make it self-retaining, the strategic location of the FC balloon in the rigid confines of the abdominal musculature inherently prevents its over-inflation and the associated life threatening morbidity like its peristaltic antegrade propulsion leading to intestinal obstruction and local pressure-necrosis of the bowel as aptly reported by Purewal and collegues. ${ }^{6}$

For preventing dumping syndrome, the route and the timings of semisolids (through RT) may simply be separated from that of the liquids (through FC). With experience, the authors have tailored to the present calibre of the RT not only for effortless infusion of semisolids but also to circumvent dumping syndrome due to inadvertent rapid infusion. As routinely practised in the authors' surgical unit, thoroughly smashed homecooked food could very well be administered (instead of costly commercial preparations) even by the patient's relatives after adequate training. The authors have adopted this policy given that such food could easily be modified as per the ethnicity and geographical location of the patients. These issues might especially be of great concern to resource-poor countries.

In cases of tube-malfunction, these could comfortably and promptly be replaced. If required, the entire PJ effluent may be infused directly through the RT without filtering it as deemed necessary by other clinicians. ${ }^{4}$

It does not add to the list of the potential complications related to the stoma. In fact, local stoma therapist and the nursing staff also found it user-friendly and appreciated it well. It is easy to learn even by the most junior member of the surgical team and the local paramedics, alike. Lastly, it not only recruits the otherwise defunct distal bowel segment (MF) for digestion/ absorption of nutrients but also has remarkable trophic effects on it as reported by other researchers. ${ }^{1}$ Overall, this has positive clinical impact on patients with surgically created short bowel syndrome. ${ }^{4}$

However, the authors have observed just one limitation of this technique - there seems to be a bleak margin of error for precisely asserting the plane of rent in the abdominal musculature. Inability to do so, annuls the entire procedure in its early course. Therefore, as this judgment has a subtle component of subjectivity, the authors always encourage postgraduates to master this key step right in the beginning of their learning curve (which was 5 cases in authors' institute) to avoid inadvertent failures. Till now, the authors have successfully used this method in 18 cases without any complications and could avoid total parenteral nutrition in all of them. These patients are under regular follow-up and are doing well. Whilst, 16 have already undergone stoma reversals, two are waiting for their turn. 


\section{CONCLUSION}

The Jategaonkar technique is feasible, safe, easy-tolearn and likely cost-effective method of enteral feeding for patients with short bowel syndrome; and hence, could be recommended for wider usage.

\section{AUTHORS' CONTRIBUTION:}

PAJ, SPY: Drafted and finalised the manuscript to be published.

\section{CONFLICT OF INTEREST:}

Authors declared no conflict of interest.

\section{REFERENCES}

1. Schörghuber M, Fruhwald S. Effects of enteral nutrition on gastrointestinal function in patients who are critically ill. Lancet Gastroenterol Hepatol 2018; 3:281-7.
2. Nagar A, Mehrotra S, Yadav A, Mangla V, Lalwani S, Mehta N, et al. Distal bowel re-feeding in patients with proximal jejunostomy. J Gastrointest Surg 2018; 22:1251-7.

3. Drenckpohl D, Vegunta R, Knaub L, Holterman M, Wang H, Macwan $\mathrm{K}$, et al. Reinfusion of succus entericus into the mucous fistula decreases dependence on parenteral nutrition in neonates. ICAN: Infant Child \& Adolescent Nutrition 2012; 4:168-74.

4. Liu MY, Tang HC, Yang HL, Chang SJ. Is jejunostomy output nutrient or waste in short bowel syndrome? Experience from six cases. Asia Pac J Clin Nutr 2016; 25:430-5.

5. Dash NR, Singh AN, Kilambi R. Balloon-inflated catheters for enteral feeding: A word of caution. Indian J Surg 2018; 80: 14-8.

6. Purewal $\mathrm{P}$, Malik $\mathrm{H}$, Pettit $\mathrm{S}$. The importance of monitoring feeding tubes in mucous fistulae. BMJ Case Rep 2013; 2013: bcr2012008424.

\section{..........}

